\title{
Validação do arranjo espacial do percevejo-do-colmo em arroz irrigado por inundação
}

\author{
Spatial arrangement validation of the rice stem bug in flooded rice field
}

Juliano de Bastos Pazini ${ }^{1}$, Enio Júnior Seidel ${ }^{2}$, Fernando Felisberto da Silva ${ }^{3}$, José Alexandre Freitas

Barrigossi ${ }^{4}$, José Francisco da Silva Martins ${ }^{5}$ e Robson Antonio Botta ${ }^{1}$

\author{
${ }^{1}$ Universidade Federal de Pelotas, Pelotas, RS, Brasil \\ julianopazzini@hotmail.com; robson_a.b@hotmail.com \\ ${ }^{2}$ Universidade Federal de Santa Maria, Santa Maria, RS, Brasil \\ enioseidel@gmail.com \\ ${ }^{3}$ Universidade Federal do Pampa, Itaqui, RS, Brasil \\ fernando.silva@unipampa.edu.br \\ ${ }^{4}$ Empresa Brasileira de Pesquisa Agropecuária (EMBRAPA Arroz e Feijão), Santo Antônio de Goiás, GO, Brasil \\ jose.barrigossi@embrapa.br \\ ${ }^{5}$ Empresa Brasileira de Pesquisa Agropecuária (EMBRAPA Clima Temperado), Pelotas, RS, Brasil \\ jose.martins@embrapa.br
}

\section{Resumo}

Com o objetivo de validar o padrão de arranjo espacial de Tibraca limbativentris Stal, 1860 (Hemiptera: Pentatomidae) na cultura do arroz irrigado por inundação na região do Planalto da Campanha do Rio Grande do Sul, realizou-se estudo em lavouras de arroz irrigado por inundação no município de Itaqui-RS, nas safras de 2009/10, $2010 / 11$ e 2011/12. Para execução das amostragens, estabeleceram-se grids regulares georreferenciados, totalizando 81 pontos espaçados em 50 m na safra 2009/10, 693 na safra 2010/11 e 352 na safra 2011/12, ambas com pontos equidistantes $\sim 10 \mathrm{~m}$. Sobre cada ponto dos grids regulares foi realizada a amostragem de adultos e ninfas do inseto. Efetuou-se a análise estatística descritiva para as variáveis adultos, ninfas e adultos + ninfas por meio do cálculo da média, desvio padrão e valores mínimo e máximo e dos índices de dispersão: razão variância/média, indice de Morisita, índice $k$, coeficiente de Green e potência de Taylor. O arranjo espacial do percevejo-do-colmo foi validado quando os índices de dispersão apresentaram resultados similares. O arranjo espacial do percevejo-do-colmo T. limbativentris em arroz irrigado por inundação é do tipo agregado, independentemente do nível de infestação e da fase de vida do inseto.

Palavras-chave: Tibraca limbativentris; Distribuição; Oryza sativa

\section{Abstract}

In order to spatial arrangement validation of Tibraca limbativentris Stal, 1860 (Hemiptera: Pentatomidae) in flooded rice field located at the "Planalto da Campanha" Region, Rio Grande do Sul (RS), Brazil, was carried out a study in flooded rice fields at Itaqui, Rio Grande do Sul State, Brazil, in the 2009/10, 2010/11 and 2011/12 harvests. Were established georeferenced grids for the samplings, totaling 81 points spaced $\sim 50 \mathrm{~m}$ in the 2009/10 harvest and, 693 and 352 points in the 2010/11 and 2011/12 harvests, respectively, both with equidistant points $\sim 10 \mathrm{~m}$. Adults and nymphs samplings were performed on each point of the regular grids. We conducted a descriptive statistical analysis for the adults, nymphs and adults + nymphs variables by calculations the mean, standard deviation and minimum and maximum values and dispersion indices: variance/mean, Morisita index, $k$ index, Green coefficient and Taylor potency. The rice stem bug spatial arrangement has been validated when the dispersion indices showed similar results. The rice stem bug T. limbativentris spatial arrangement in flooded rice is the aggregate type, regardless of the infestation level and life stage of the insect.

Keywords: Tibraca limbativentris; Distribution; Oryza sativa 


\section{Introdução}

O arroz (Oryza sativa L.) é um componente básico da alimentação humana no Brasil, sendo que cerca de $60 \%$ da produção nacional provém de cultivos irrigados por inundação no estado do Rio Grande do Sul (CONAB, 2015). Praticamente 30\% das lavouras se localizam no Planalto da Campanha do Estado, onde o percevejo-do-colmo Tibraca limbativentris Stal, 1860 (Hemiptera: Pentatomidae) se destaca como um fator biótico prejudicial à cultura. Trata-se de um inseto-praga que danifica as plantas nas fases vegetativa e reprodutiva, provocando os sintomas de "coração morto" e "panícula branca", respectivamente (REUNIÃO, 2014).

A distribuição dos organismos no espaço é uma característica ecológica da espécie, influenciada pela natalidade, mortalidade e migração (TOLEDO et al., 2006), sendo a distribuição espacial a forma como os indivíduos de uma população se dispersam em seu habitat. O modelo de dispersão no habitat varia entre as espécies e entre as populações da mesma espécie. Essa variação pode estar associada a diversos fatores, tais como, locais para hibernação, postura, temperatura, umidade, proteção do ataque de predadores e abundância de alimento (RABINOVICH, 1980).

Tratando-se do Manejo Integrado de Pragas (MIP), um padrão de amostragem que não coincide com o padrão de distribuição espacial do inseto pode resultar em erros na estimativa da população da praga em programas de amostragem (SILVA et al., 2014a). Estimativas das populações são necessárias para compreender a dinâmica da população de uma espécie-praga e para fornecer informações importantes para subsidiar a tomada de decisão no controle (COSTA et al., 2007). O arranjo espacial de populações de insetos enquadra-se em padrões que podem ser do tipo aleatório, uniforme ou agregado (RABINOVICH, 1980). A determinação desses padrões de arranjo pode ser obtida por meio de índices de dispersão, que permitem inferir sobre a etologia da espécie (REAY-JONES et al., 2010), aperfeiçoar os sistemas de amostragem (SILVA et al., 2014b) e, consequentemente, o manejo integrado da espéciepraga (MELO et al., 2006).

Em trabalho pioneiro sobre distribuição do percevejo-do-colmo em lavouras de arroz irrigado, realizado por Costa e Link (1992), o levantamento populacional foi baseado apenas num reduzido número de amostras e somente duas fases do ciclo da cultura. Além disso, não foram consideradas as distâncias e localizações dos pontos amostrados. Considerando o avanço das tecnologias de cultivo, bem como o surgimento de novas cultivares surge a necessidade de estudos que incluam um maior número de amostras e épocas de amostragem. Torna-se necessário, ainda, que mais de um índice de dispersão seja estudado antes de se inferir com maior segurança sobre a distribuição espacial de uma determinada espécie de inseto (RABINOVICH, 1980).

O objetivo deste trabalho foi de validar o padrão de arranjo espacial de T. limbativentris na cultura do arroz irrigado por inundação na região do Planalto da Campanha do Rio Grande do Sul, por meio da análise de diferentes índices de dispersão utilizados na literatura em estudos sobre a distribuição de insetos.

\section{Material e métodos}

O experimento foi realizado nos anos agrícolas de 2009/10, 2010/11 e 2011/12 em lavoura comercial de arroz irrigado por inundação pertencente à Fazenda Pitangueira - Unidade Cambaí, município de Itaqui-RS, localizada a $2^{\circ} 09^{\prime} 56.52^{\prime \prime S}$ e $56^{\circ} 29^{\prime} 20.06^{\prime \prime} \mathrm{W}$. O solo do local é classificado como Luvissolo Crômico Pálico abrúptico plíntico, com declividade média de 4,8\%. O clima da região, conforme classificação de Köppen-Geiger é do tipo "Cfa", isto é, subtropical, temperado quente, apresentando chuvas bem distribuídas e estações bem definidas, com temperatura média anual entre 19,6 a $20^{\circ} \mathrm{C}$ e precipitação média anual entre 1.537 a $1.659 \mathrm{~mm}$. 
Nos anos de estudo, a cultura foi implantada no sistema de cultivo mínimo, com a semeadura realizada na primeira semana do mês de outubro, com a cultivar IRGA 417 na densidade de 60 sementes por metro linear num espaçamento de 0,17 metros entre linhas. Após análise química do solo, na adubação de base, foram aplicados 286 $\mathrm{kg} \mathrm{ha}^{-1}$ da formulação 04-17-27 (N-P-K) no sulco de semeadura. A adubação em cobertura foi realizada com $150 \mathrm{~kg}$ $\mathrm{ha}^{-1}$ de 45-00-00 (N-P-K) aos 15 dias após a emergência, antes da entrada de água e; $75 \mathrm{~kg} \mathrm{ha}^{-1}$ de 30-00-20 (N-P-K) na diferenciação do primórdio floral. O manejo fitossanitário foi efetuado, sempre que necessário, de acordo com as recomendações técnicas da pesquisa para a cultura do arroz irrigado (REUNIÃO, 2014), porém, sem aplicações de inseticidas. Após a colheita do arroz, que ocorreu em meados de fevereiro, a área de cultivo foi manejada com duas passadas de grade aradora mais uma passada de grade niveladora para incorporação dos restos culturais (resteva), porém, na safra de 2011/12, o manejo da área e destruição da resteva foi realizado com rolo-faca.

(a)

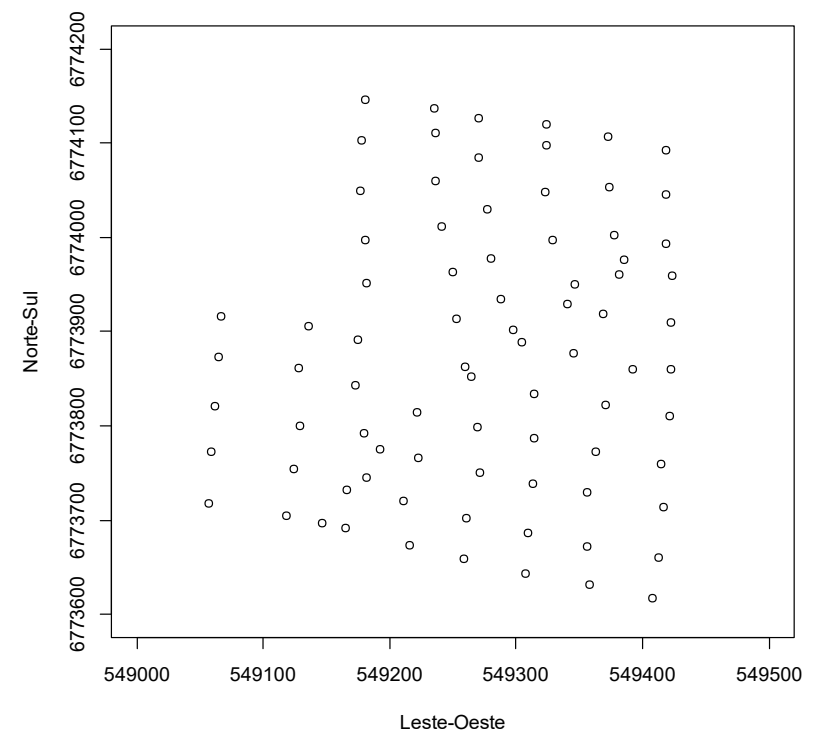

(b)

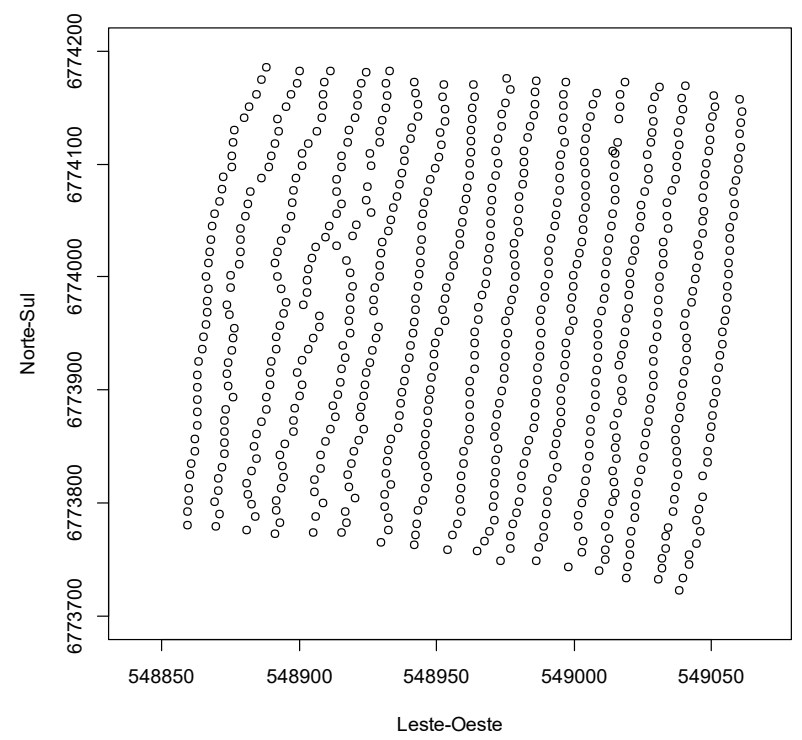

(c)

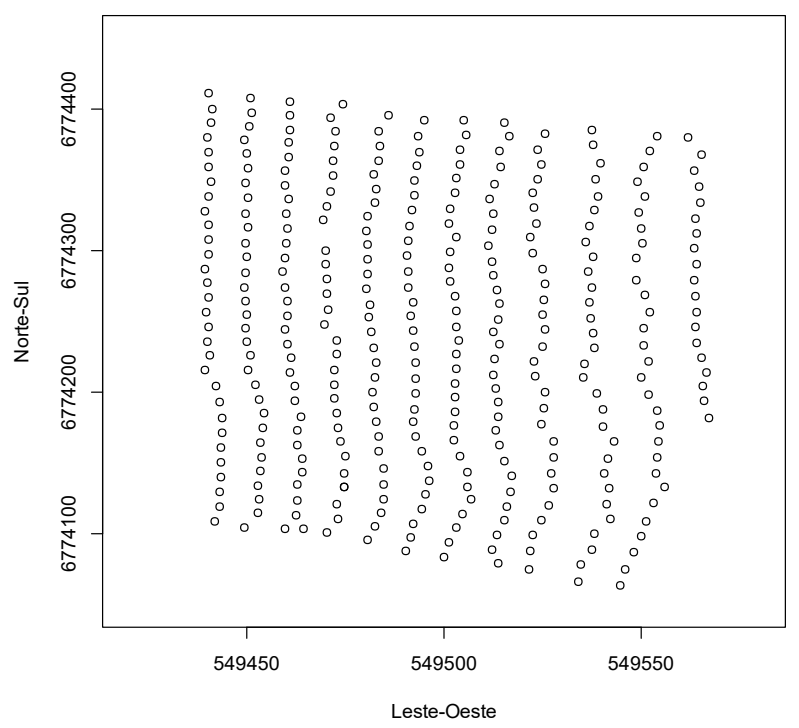

Figura 1 - Grades de amostras na área de estudo, contendo: (a) 81 pontos de amostragem georreferenciados com espaçamento de 50 x 50 m na safra 2009/10; (b) 693 pontos de amostragem georreferenciados com espaçamento de 10 x 10 m na safra 2010/11; (c) 352 pontos de amostragem georreferenciados com espaçamento de 10 x $10 \mathrm{~m}$ na safra 2011/12 
O plano de amostragem de indivíduos de T. limbativentris foi estabelecido seguindo uma grade mais ou menos regular previamente estabelecido e as coordenadas geográficas de cada ponto de amostragem foi obtida com aparelho GPS (Garmin eTrex ${ }^{\circledR}$ 30). As distâncias entre os pontos de amostragem foram: 50 m na safra $2009 / 10$ e 10 $\mathrm{m}$ nas safras 2010/11 e 2011/12 (Figuras 1 a, b e c). Cada unidade de amostra correspondeu a 0,25 $\mathrm{m}^{2}$, demarcada com uma estrutura metálica medindo $0,5 \mathrm{~m}$ x $0,5 \mathrm{~m}$, que foi posicionada no ponto de amostragem. Todas as plantas dentro do quadro foram rigorosamente examinadas e os números de adultos e ninfas de T. limbativentris foram contabilizados.

O monitoramento compreendeu amostragens quinzenais em distintos estádios fenológicos das plantas de arroz e pós-colheita, uma vez que o inseto pode se manter entre a resteva, principalmente em condições de cultivo mínimo (PAZINI et al., 2012; BOTTA et al., 2014). Na safra 2009/10, o campo foi amostrado três vezes, sendo a primeira no dia 06/01/2010, quando as plantas encontravam-se no estádio fenológico V11; a segunda no dia 02/02/2010 no estádio R6 e; a última em 08/03/2010 na resteva não incorporada, sendo obtidas 81 amostras em cada amostragem. Em 2010/11, o monitoramento abrangeu cinco ocasiões de amostragem, nas seguintes datas e fases da cultura: 13/12/2010 quando as plantas encontravam-se em estádio fenológico V8; 27/12/2010 em estádio V10/V11; 18/01/2011 em estádio R1; 12/02/2011 em estádio R9 e; 15/02/201, na resteva não incorporada, num total de 693 amostras em cada ocasião. Por fim, na safra 2011/12, monitorou-se em oito ocasiões, nas seguintes datas e fases da cultura: 19/11/2011 quando as plantas encontravam-se em estádio fenológico V4; 03/12/2011 em estádio V6; 17/12/2011 em estádio V8/V9; 07/01/2012 em estádio V11; 21/01/2012 em estádio R1; 02/02/2012 em estádio R5; 15/02/2012 em estádio R9 e; 29/02/2012 na resteva incorporada com rolo-faca, totalizando 352 amostras cada. Os estádios fenológicos das plantas de arroz em cada período amostral foram determinados segundo escala de Counce et al. (2000). As demais especificidades metodológicas adotadas em cada safra estão apresentadas na Tabela 1.

Tabela 1 - Descrição da metodologia adotada em cada safra para o estudo da distribuição espacial do percevejo-docolmo na cultura do arroz irrigado por inundação. Itaqui-RS

\begin{tabular}{|c|c|c|c|c|c|}
\hline \multirow{2}{*}{ Safra } & \multirow{2}{*}{ Área (ha) } & \multicolumn{2}{|c|}{ Pontos amostrais } & \multicolumn{2}{|r|}{ Amostragens } \\
\hline & & Número/período amostral & Equidistância (m) & Número/safra & Período amostral \\
\hline 2009/10 & 13,50 & 81 & 50 & 3 & I; II; III \\
\hline 2010/11 & 7,67 & 693 & 10 & 5 & IV; V; VI; VII; VIII \\
\hline 2011/12 & 4,00 & 352 & 10 & 8 & IX; X; XI; XII; XIII; XIV; XV; XVI \\
\hline
\end{tabular}

${ }^{\mathrm{I}} \mathrm{V} 11 ;{ }^{\mathrm{II}} \mathrm{R} 6 ;{ }^{\mathrm{II}}$ resteva não incorporada; ${ }^{\mathrm{IV}} \mathrm{V} 8 ;{ }^{\mathrm{V}} \mathrm{V} 10 / \mathrm{V} 11 ;{ }^{\mathrm{VI}} \mathrm{R} 1 ;{ }^{\mathrm{VII}} \mathrm{R} 9 ;{ }^{\mathrm{VIII}}$ resteva não incorporada; ${ }^{\mathrm{IX}} \mathrm{V} 4 ;{ }^{\mathrm{x}} \mathrm{V} 6 ;{ }^{\mathrm{XI}} \mathrm{V} 8 / \mathrm{V} 9 ;{ }^{\mathrm{XII}} \mathrm{V} 11 ;{ }^{\mathrm{XII}} \mathrm{R} 1 ;{ }^{\mathrm{XIV}} \mathrm{R} 5{ }^{\mathrm{XV}} \mathrm{R} 9$; ${ }^{\mathrm{XVI}}$ resteva incorporada com o implemento agrícola rolo-faca. Estádios conforme Counce et al. (2000).

Nas safras 2010/11 e 2011/12, verificou-se a necessidade da redução no espaçamento entre pontos e o aumento no número de amostras e épocas de amostragem, a fim de se identificar com maior exatidão o comportamento da população por unidade de área nos distintos estádios fenológicos das plantas de arroz e validar o arranjo espacial do percevejo-do-colmo. Apesar das modificações metodológicas realizadas, a representatividade amostral (número de pontos e distância entre pontos) para o estudo foi mantida, estando em conformidade com as recomendações para o monitoramento do inseto (REUNIÃO, 2014; ALVES et al., 2016).

Procedeu-se a análise estatística descritiva para as variáveis adultos, ninfas e adultos+ninfas com o cálculo da média, desvio padrão e valores mínimo e máximo e dos índices de dispersão: razão variância/média, índice de Morisita, 
índice $\mathrm{k}$, coeficiente de Green e potência de Taylor, índices frequente e recentemente utilizados em estudos sobre a dispersão de insetos-pragas (COSTA et al., 2006; COSTA et al., 2010; DINARDO-MIRANDA et al., 2007; MARTINS et al., 2012; NICKELE et al., 2010; RIBEIRO et al., 2006; RODRIGUES et al., 2010; SOUZA et al., 2013). Utilizou-se o pacote vegan (OKSANEN et al., 2015) do software estatístico R (R DEVELOPMENT CORE TEAM, 2011) para a execução das análises.

O índice variância/média (I) foi dado pela equação (1).

$$
I=\frac{S^{2}}{m}
$$

Em que: $\mathrm{S}^{2}$ é a variância amostral e m é a média amostral.

Para este índice os valores iguais à unidade indicam disposição espacial ao acaso ou aleatória; já os valores menores indicam que a unidade aponta disposição espacial regular ou uniforme, e os valores significativamente maiores que 1 mostram disposição agregada ou contagiosa (RABINOVICH, 1980).

O índice de Morisita (I $\delta$ ) foi dado por (2).

$$
I \delta=\frac{N\left(\sum_{i=1}^{N} X_{i}^{2}-\sum_{i=1}^{N} X_{i}\right)}{\left(\sum_{i=1}^{N} X_{i}\right)^{2}-\sum_{i=1}^{N} X_{i}}
$$

Em que: $\mathrm{N}$ é o tamanho da amostra e $\mathrm{X}_{\mathrm{i}}$ é o número de insetos na i-ésima unidade amostral.

Este índice é relativamente independente da média e do número de amostras. Assim, quando I $\delta=1$ a distribuição é aleatória; I $\delta>1$ a distribuição é do tipo agregada e quando $I \delta<1$ indica uma distribuição uniforme (SILVEIRA NETO et al., 1976).

O Parâmetro k foi dado pela equação (3).

$$
k=\frac{m^{2}}{s^{2}-m}
$$

Em que: $m$ é a média amostral e $\mathrm{S}^{2}$ é a variância amostral.

$\mathrm{O}$ índice $\mathrm{k}$ indica uma distribuição uniforme quando os valores são negativos; altamente agregada quando $\mathrm{k}$ assume valores baixos e positivos $(0<\mathrm{k}<2)$; agregação moderada quando valores de k variam de 2 a 8 e; disposição aleatória quando os valores são superiores a $8(\mathrm{k}>8)($ ELLIOTT, 1979).

O Coeficiente de Green (Cx) é dado pela equação (4).

$$
C x=\frac{\left(\frac{s^{2}}{m}\right)-1}{\sum_{x}-1}
$$

Em que: $\mathrm{S}^{2}$ é a variância amostral, $\mathrm{m}$ é a média amostral e $\Sigma_{\mathrm{x}}$ é o somatório do número de indivíduos nas amostras.

O Coeficiente de Green é fundamentado na razão variância/média da distribuição. Valores negativos demostram um padrão uniforme e valores positivos um padrão de agregação; valores iguais a zero representam uma distribuição aleatória (GREEN, 1966). 
A lei da Potência de Taylor (b) baseia-se na relação existente entre a variância e a média amostral e é dada pela equação (5).

$$
S^{2}=a m^{b}
$$

Em que: a é um fator escalar, $\mathrm{S}^{2}$ é a variância, $\mathrm{m}$ é a média amostral e b é o índice de dispersão.

Quando o coeficiente b é significativamente maior que a unidade a distribuição espacial é agregada; quando é igual ou próximo à unidade o padrão é aleatório e; quando o coeficiente for menor que a unidade a distribuição é uniforme (TAYLOR, 1961). O parâmetro b foi testado pelo teste t de "Student", onde se utilizou o logaritmo (6).

$$
\log S^{2}=\log a+\mathrm{b} \log m
$$

Para revisão e validação do arranjo espacial de $T$. limbativentris (adultos, ninfas e adultos + ninfas) foi estabelecido, como critério de decisão, que o padrão de distribuição deve ser definido quando os diferentes índices estimarem resultados similares (MYERS, 1978).

\section{Resultados e discussão}

A análise da estatística descritiva para as safras 2009/10, 2010/11 e 2011/12 mostrou que os dados de adultos, ninfas e adultos+ninfas de percevejo-do-colmo apresentaram elevada variabilidade, com valores do desvio padrão maiores que a média em todas as ocasiões de amostragens (Tabelas 2, 3 e 4). Isso também foi verificado por meio do índice I, com valores maiores que a unidade, indicando alta dispersão por comportamentos de agregação (Tabelas 2, 3 e 4). Tal fato ocorreu em virtude da maior frequência de amostras com muitos e poucos insetos em zonas da lavoura. Chiba et al. (2010) obtiveram um resultado semelhante com dados de contagens, ao estudarem a variabilidade espacial e temporal de plantas daninhas, atribuindo o fato pela existência de zonas com baixa ocorrência da praga e outras com expressiva população. A mesma razão pode ser atribuída como componente explicativo para o comportamento de elevada variabilidade no presente estudo (Tabelas 2,3 e 4 ).

Observou-se, de modo geral, um aumento inicial no número médio de percevejos-do-colmo no decorrer das amostragens e, posterior redução na amostragem após a colheita, tanto para adultos, ninfas e adultos+ninfas (Tabelas 2 , 3 e 4). Os dados evidenciaram os períodos entre V4 a R4 como as épocas de maior ocorrência do percevejo-do-colmo, estádios correspondentes à época que vai do início do perfilhamento até a floração na cultura do arroz (COUNCE et al., 2000).

A população de adultos de $T$. limbativentris (Tabela 2) foi baixa no início da fase vegetativa da cultura $(13 / 12 / 2010: \mathrm{m}=0,09 ; 27 / 12 / 2010: \mathrm{m}=0,04 ; 19 / 11 / 2011: \mathrm{m}=0,05 ; 03 / 12 / 2011: \mathrm{m}=0,10)$, formada apenas por indivíduos hibernantes ou alojados na vegetação espontânea das margens da lavoura. À medida que o ciclo fenológico do arroz avançou, acentuou-se $(18 / 01 / 2011: \mathrm{m}=0,54 ; 12 / 12 / 2011: \mathrm{m}=0,66 ; 17 / 12 / 2011: \mathrm{m}=0,23 ; 02 / 02 / 2012: \mathrm{m}=$ 0,78; 15/02/2012: $\mathrm{m}=0,50)$, provavelmente, em virtude do deslocamento completo dos insetos do sítio de hibernação para a lavoura e do desenvolvimento de novas gerações da praga (COSTA; LINK, 1992). Botta et al. (2014), em estudo da flutuação populacional do percevejo-do-colmo, verificaram que o abandono total do local de hibernação ocorreu até o mês de janeiro. 
Tabela 2 - Análise estatística descritiva com valores mínimo (Mín) e máximo (Máx), média \pm desvio padrão (m \pm S) e índices de dispersão variância/média (I), Morisita (I $\delta$ ), parâmetro $\mathrm{k}$ da distribuição binomial negativa (k), coeficiente de Green $\left(\mathrm{C}_{\mathrm{X}}\right)$ e potência de Taylor $(\mathrm{b})$ para a variável "adultos" de Tibraca limbativentris na cultura do arroz irrigado por inundação. Safras 2009/10, 2010/11 e 2011/12. Itaqui-RS.

\begin{tabular}{|c|c|c|c|c|c|c|c|c|}
\hline \multirow{2}{*}{$\begin{array}{l}\text { Período amostral }^{\#} \\
\text { (data-estádio fenológico) }\end{array}$} & \multirow{2}{*}{ Mín } & \multirow{2}{*}{ Máx } & \multirow{2}{*}{$\mathbf{m} \pm \mathbf{S}$} & \multicolumn{5}{|c|}{ Índices de dispersão } \\
\hline & & & & (I) & (Iס) & (k) & $\left(\mathbf{C}_{\mathbf{x}}\right)$ & (b) \\
\hline \multicolumn{9}{|c|}{ Safra 2009/10 } \\
\hline $06 / 01 / 2010$ - V11 & 0,000 & 5,000 & $0,750 \pm 0,990$ & 1,310 & $1,410^{*}$ & 2,410 & 0,005 & \multirow{3}{*}{$0,890^{\mathrm{n}}$} \\
\hline 02/02/2010 - R6 & 0,000 & 3,000 & $0,380 \pm 0,710$ & 1,340 & $1,910^{*}$ & 1,110 & 0,010 & \\
\hline 08/03/2010 - RN ${ }^{\mathrm{I}}$ & 0,000 & 6,000 & $0,490 \pm 0,950$ & 1,820 & $2,700^{*}$ & 0,590 & 0,020 & \\
\hline \multicolumn{9}{|c|}{ Safra 2010/11 } \\
\hline $13 / 12 / 2010$ - V8 & 0,000 & 3,000 & $0,090 \pm 0,330$ & 1,160 & $2,670^{*}$ & 0,600 & 0,002 & \multirow{5}{*}{$1,230^{*}$} \\
\hline 27/12/201 - V10/V11 & 0,000 & 3,000 & $0,040 \pm 0,250$ & 1,490 & $12,810^{*}$ & 0,080 & 0,010 & \\
\hline 18/01/2011 - R1 & 0,000 & 30,000 & $0,540 \pm 1,490$ & 4,120 & $6,770^{*}$ & 0,170 & 0,008 & \\
\hline 12/02/2011 - R9 & 0,000 & 4,000 & $0,660 \pm 1,000$ & 1,510 & $1,780^{*}$ & 1,280 & 0,001 & \\
\hline $15 / 02 / 2011-R^{I}$ & 0,000 & 4,000 & $0,080 \pm 0,350$ & 1,450 & $6,510^{*}$ & 0,180 & 0,007 & \\
\hline \multicolumn{9}{|c|}{ Safra 2011/12 } \\
\hline 19/11/2011 - V4 & 0,000 & 4,000 & $0,050 \pm 0,320$ & 1,790 & $15,080^{*}$ & 0,070 & 0,040 & \multirow{8}{*}{$1,080^{*}$} \\
\hline 03/12/2011 - V6 & 0,000 & 4,000 & $0,100 \pm 0,480$ & 1,510 & $4,420^{*}$ & 0,290 & 0,009 & \\
\hline 17/12/2011 - V8/V9 & 0,000 & 4,000 & $0,230 \pm 0,510$ & 1,140 & $1,620^{*}$ & 1,600 & 0,001 & \\
\hline 07/01/2012 - V11 & 0,000 & 10,000 & $0,110 \pm 0,640$ & 3,490 & $22,480^{*}$ & 0,040 & 0,060 & \\
\hline 21/01/2012 - R1 & 0,000 & 2,000 & $0,090 \pm 0,310$ & 1,020 & $1,330^{\mathrm{ns}}$ & 3,080 & 0,001 & \\
\hline 02/02/2012 - R5 & 0,000 & 6,000 & $0,780 \pm 1,190$ & 1,800 & $2,030^{*}$ & 0,970 & 0,002 & \\
\hline 15/02/2012 - R9 & 0,000 & 4,000 & $0,500 \pm 0,860$ & 1,460 & $1,940^{*}$ & 1,060 & 0,002 & \\
\hline 29/02/2012 - RI II & 0,000 & 1,000 & $0,002 \pm 0,050$ & 1,000 & -- & -- & -- & \\
\hline
\end{tabular}

Resteva não incorporada; "Resteva incorporada com o implemento agrícola rolo-faca. ${ }^{\#}$ Estádios fenológicos conforme Counce et al. (2000). *Significativo ao nível de $5 \%$ de probabilidade; ${ }^{\text {ns }}$ Não significativo ao nível de $5 \%$ de probabilidade; -- Quantidade de insetos adultos insuficiente para o cálculo.

Tabela 3 - Análise estatística descritiva com valores mínimo (Mín) e máximo (Máx), média \pm desvio padrão (m \pm S) e índices de dispersão variância/média (I), Morisita (I $\delta$ ), parâmetro k da distribuição binomial negativa (k), coeficiente de Green $\left(\mathrm{C}_{\mathrm{X}}\right)$ e potência de Taylor (b) para a variável "ninfas" de Tibraca limbativentris na cultura do arroz irrigado por inundação. Safras 2009/10, 2010/11 e 2011/12. Itaqui-RS.

\begin{tabular}{|c|c|c|c|c|c|c|c|c|}
\hline \multirow{2}{*}{$\begin{array}{l}\text { Período amostral }^{\#} \\
\text { (data-estádio fenológico) }^{\text {(a) }}\end{array}$} & \multirow{2}{*}{ Mín } & \multirow{2}{*}{ Máx } & \multirow{2}{*}{$\mathbf{m} \pm \mathbf{S}$} & \multicolumn{5}{|c|}{ Índices de dispersão } \\
\hline & & & & (I) & (ID) & (k) & $\left(\mathbf{C}_{\mathrm{x}}\right)$ & (b) \\
\hline \multicolumn{9}{|c|}{ Safra 2009/10 } \\
\hline 06/01/2010 - V11 & 0,000 & 12,000 & $0,960 \pm 2,000$ & 4,190 & $4,310^{*}$ & 0,300 & 0,040 & \multirow{3}{*}{$1,470^{\mathrm{ns}}$} \\
\hline 02/02/2010 - R6 & 0,000 & 9,000 & $1,230 \pm 2,080$ & 3,500 & $3,020^{*}$ & 0,490 & 0,020 & \\
\hline 08/03/2010 - RN ${ }^{\mathrm{I}}$ & 0,000 & 4,000 & $0,290 \pm 0,760$ & 1,970 & $4,400^{*}$ & 0,300 & 0,040 & \\
\hline \multicolumn{9}{|c|}{ Safra 2010/11 } \\
\hline $13 / 12 / 2010-V 8$ & 0,000 & 35,000 & $0,590 \pm 2,450$ & 10,080 & $16,220^{*}$ & 0,060 & 0,020 & \multirow{5}{*}{$1,910^{*}$} \\
\hline 27/12/201 - V10/V11 & 0,000 & 46,000 & $0,940 \pm 3,100$ & 10,220 & $10,750^{*}$ & 0,100 & 0,010 & \\
\hline 18/01/2011 - R1 & 0,000 & 29,000 & $0,610 \pm 1,430$ & 3,370 & $4,880^{*}$ & 0,250 & 0,005 & \\
\hline 12/02/2011 - R9 & 0,000 & 24,000 & $0,760 \pm 1,350$ & 2,390 & $2,820^{*}$ & 0,540 & 0,002 & \\
\hline $15 / 02 / 2011-\mathrm{RN}^{\mathrm{I}}$ & 0,000 & 7,000 & $0,340 \pm 0,940$ & 2,550 & $5,510^{*}$ & 0,220 & 0,006 & \\
\hline
\end{tabular}


Tabela 3 - Análise estatística descritiva com valores mínimo (Mín) e máximo (Máx), Continuação...

\begin{tabular}{|c|c|c|c|c|c|c|c|c|}
\hline \multirow{2}{*}{$\begin{array}{l}\text { Período amostral }^{\#} \\
\text { (data-estádio fenológico) }\end{array}$} & \multirow{2}{*}{ Mín } & \multirow{2}{*}{ Máx } & \multirow{2}{*}{$\mathbf{m} \pm \mathbf{S}$} & \multicolumn{5}{|c|}{ Índices de dispersão } \\
\hline & & & & (I) & (ID) & (k) & $\left(C_{x}\right)$ & (b) \\
\hline \multicolumn{9}{|c|}{ Safra 2011/12 } \\
\hline 19/11/2011 - V4 & 0,000 & 0,000 & -- & -- & -- & -- & -- & \multirow{8}{*}{$1,330^{*}$} \\
\hline 03/12/2011 - V6 & 0,000 & 0,000 & -- & -- & -- & -- & -- & \\
\hline 17/12/2011 - V8/V9 & 0,000 & 2,000 & $0,010 \pm 0,130$ & 1,480 & $58,660^{*}$ & 0,020 & 0,160 & \\
\hline 07/01/2012 - V11 & 0,000 & 18,000 & $1,030 \pm 2,080$ & 4,180 & $4,080^{*}$ & 0,320 & 0,008 & \\
\hline 21/01/2012 - R1 & 0,000 & 26,000 & $1,120 \pm 2,420$ & 5,220 & $4,740^{*}$ & 0,260 & 0,010 & \\
\hline 02/02/2012 - R5 & 0,000 & 35,000 & $0,490 \pm 2,390$ & 11,490 & $22,170^{*}$ & 0,040 & 0,060 & \\
\hline 15/02/2012 - R9 & 0,000 & 48,000 & $1,000 \pm 4,850$ & 23,480 & $23,350 *$ & 0,040 & 0,060 & \\
\hline 29/02/2012 - RII & 0,000 & 3,000 & $0,010 \pm 0,160$ & 2,490 & $176,000 *$ & 0,007 & 0,490 & \\
\hline
\end{tabular}

Resteva não incorporada; "Resteva incorporada com o implemento agrícola rolo-faca. "Estádios fenológicos conforme Counce et al. (2000). *Significativo ao nível de $5 \%$ de probabilidade; ${ }^{\text {ns }}$ Não significativo ao nível de $5 \%$ de probabilidade; -- Quantidade de insetos adultos insuficiente para o cálculo.

Tabela 4 - Análise estatística descritiva com valores mínimo (Mín) e máximo (Máx), média \pm desvio padrão (m $\pm \mathrm{S}$ ) e índices de dispersão variância/média (I), Morisita (IS), parâmetro $\mathrm{k}$ da distribuiç̧ão binomial negativa (k), coeficiente de

Green $\left(\mathrm{C}_{\mathrm{X}}\right)$ e potência de Taylor (b) para a variável "adultos+ninfas" de Tibraca limbativentris na cultura do arroz irrigado por inundação. Safras 2009/10, 2010/11 e 2011/12. Itaqui-RS.

\begin{tabular}{|c|c|c|c|c|c|c|c|c|}
\hline \multirow{2}{*}{$\begin{array}{l}\text { Período amostral }^{\#} \\
\text { (data-estádio fenológico) }^{\text {(a) }}\end{array}$} & \multirow{2}{*}{ Mín } & \multirow{2}{*}{ Máx } & \multirow{2}{*}{$\mathbf{m} \pm \mathbf{S}$} & \multicolumn{5}{|c|}{ Índices de dispersão } \\
\hline & & & & (I) & (ID) & (k) & $\left(\mathbf{C}_{\mathbf{x}}\right)$ & (b) \\
\hline \multicolumn{9}{|c|}{ Safra 2009/10 } \\
\hline 06/01/2010 - V11 & 0,000 & 12,000 & $1,720 \pm 2,290$ & 3,060 & $2,190^{*}$ & 0,830 & 0,010 & \multirow{3}{*}{$1,520 *$} \\
\hline 02/02/2010 - R6 & 0,000 & 9,000 & $1,670 \pm 2,240$ & 3,100 & $2,290^{*}$ & 0,760 & 0,010 & \\
\hline 08/03/2010 - RN ${ }^{\mathrm{I}}$ & 0,000 & 7,000 & $0,790 \pm 1,280$ & 2,080 & $2,370^{*}$ & 0,730 & 0,010 & \\
\hline \multicolumn{9}{|c|}{ Safra 2010/11 } \\
\hline 13/12/2010 - V8 & 0,000 & 35,000 & $0,700 \pm 2,480$ & 8,820 & $12,250^{*}$ & 0,090 & 0,010 & \multirow{5}{*}{$1,250^{*}$} \\
\hline 27/12/201 - V10/V11 & 0,000 & 46,000 & $0,990 \pm 3,130$ & 9,840 & $9,890 *$ & 0,110 & 0,010 & \\
\hline 18/01/2011 - R1 & 0,000 & 30,000 & $1,160 \pm 2,150$ & 4,000 & $3,600 *$ & 0,380 & 0,003 & \\
\hline 12/02/2011 - R9 & 0,000 & 24,000 & $1,430 \pm 1,730$ & 2,090 & $1,770 *$ & 1,300 & 0,001 & \\
\hline $15 / 02 / 2011-\mathrm{RN}^{\mathrm{I}}$ & 0,000 & 7,000 & $0,430 \pm 1,040$ & 2,480 & $4,410^{*}$ & 0,290 & 0,005 & \\
\hline \multicolumn{9}{|c|}{ Safra 2011/12 } \\
\hline 19/11/2011 - V4 & 0,000 & 4,000 & $0,060 \pm 0,330$ & 1,800 & $15,080^{*}$ & 0,070 & 0,040 & \multirow{8}{*}{$1,350^{*}$} \\
\hline $03 / 12 / 2011$ - V6 & 0,000 & 4,000 & $0,150 \pm 0,480$ & 1,520 & $4,430^{*}$ & 0,290 & 0,010 & \\
\hline 17/12/2011 - V8/V9 & 0,000 & 4,000 & $0,240 \pm 0,520$ & 1,140 & $1,580^{*}$ & 1,740 & 0,001 & \\
\hline 07/01/2012 - V11 & 0,000 & 18,000 & $1,150 \pm 2,160$ & 4,040 & $3,450^{*}$ & 0,370 & 0,007 & \\
\hline 21/01/2012 - R1 & 0,000 & 27,000 & $1,220 \pm 2,500$ & 5,110 & $4,360^{*}$ & 0,290 & 0,009 & \\
\hline 02/02/2012 - R5 & 0,000 & 36,000 & $1,280 \pm 2,770$ & 6,000 & $4,900^{*}$ & 0,250 & 0,010 & \\
\hline 15/02/2012 - R9 & 0,000 & 48,000 & $1,510 \pm 4,910$ & 15,980 & $10,920 *$ & 0,100 & 0,020 & \\
\hline 29/02/2012 - RI ${ }^{\mathrm{II}}$ & 0,000 & 3,000 & $0,010 \pm 0,180$ & 2,190 & $105,600 *$ & 0,010 & 0,290 & \\
\hline
\end{tabular}

Resteva não incorporada; "Resteva incorporada com o implemento agrícola rolo-faca. "Estádios fenológicos conforme Counce et al. (2000). *Significativo ao nível de $5 \%$ de probabilidade; ${ }^{\text {ns }}$ Não significativo ao nível de $5 \%$ de probabilidade; -- Quantidade de insetos adultos insuficiente para o cálculo. 
Com relação às ninfas do percevejo-do-colmo, a densidade populacional média verificada nas três safras foi comparativamente semelhante, com pequenas variações ao longo das épocas amostrais, excetuando as ocasiões de amostragem dos dias 19/11/2011 e 03/12/2011, realizadas em V4 e V6, respectivamente, onde a população foi inexistente (Tabela 3). Como a duração do período embrionário do percevejo-do-colmo varia entre 6 a 9 dias (BOTTON et al., 1996) e, que em condições naturais do ambiente de lavoura o intervalo pode ser maior, essa ausência pode ser explicada, em razão de que estes períodos correspondiam, provavelmente, a períodos de pré-oviposição e surgimento dos ovos da população de adultos pós-hibernantes presentes nestas ocasiões de amostragem, já que ninfas foram constatadas na amostragem seguinte, do dia 17/12/2011. Desta forma, a presença de ninfas foi somente observada, de modo geral, a partir dos estádios V8 e V9 da cultura do arroz $(06 / 01 / 2010: \mathrm{m}=0,96 ; 13 / 12 / 2010: \mathrm{m}=$ 0,59; 17/12/2011: $\mathrm{m}=0,01)$, indicando o desenvolvimento da primeira geração estival próximo a este período e um incremento vertiginoso da população do percevejo-do-colmo na lavoura como consequência disto (BOTTA et al., 2014).

As diminuições nas densidades populacionais de adultos, ninfas e adultos+ninfas verificadas nas últimas épocas amostrais ocorreram devido às amostragens terem sido efetuadas na resteva da cultura nas safras 2009/10 e 2010/11 e após preparo antecipado da área com a resteva incorporada por rolo-faca na safra 2011/12 (Tabelas 2, 3 e 4). Os efeitos mecânicos da colheita e de manejo de solo condicionaram alta mortalidade da população da praga presente na lavoura, conforme demonstrado por Pazini et al. (2012), bem como estimularam sua dispersão a procura de locais mais apropriados, inclusive em preparo à hibernação, que ocorre a partir do mês de março (BOTTA et al., 2014).

Os índices utilizados no estudo indicaram forte agregação para T. limbativentris. Com base nas amostragens realizadas nas três safras para as variáveis adultos, ninfas e adultos+ninfas do percevejo-do-colmo, os valores obtidos na razão variância/média (I) indicaram uma disposição espacial agregada (RABINOVICH, 1980), independentemente da época de amostragem e nível de infestação (Tabela 2, 3 e 4). Arranjo espacial semelhante também foi verificado pelo índice de Morisita (Iס) (SILVEIRA NETO et al., 1976), conforme demonstrado pelo teste de afastamento da aleatoriedade desse índice a 5\% de probabilidade, exceto para adultos na ocasião do dia 21/01/2012 (Tabela 2, 3 e 4). O índice $\mathrm{k}$ apresentou também valores dentro do intervalo de alta agregação $(0<\mathrm{k}<2)($ ELLIOTT, 1979), variando entre 0,04 e 1,60 para adultos do percevejo-do-colmo em 13 das 16 ocasiões amostrais, 0,007 e 0,54 para ninfas e entre 0,01 e 1,74 para adultos+ninfas em todas as amostragens (Tabela 2, 3 e 4). Disposição agregada para o percevejo-do-colmo foi observada ainda na análise do Coeficiente de dispersão de Green $\left(\mathrm{C}_{\mathrm{x}}\right)$, em todas as amostragens das três safras (Tabela 2, 3 e 4). Da mesma forma, o valor do índice b da lei de potência de Taylor indicou um arranjo espacial agregado para T. limbativentris para adultos e ninfas nas safras 2010/11 e 2011/12 e, para a variável adultos+ninfas, esse padrão foi constatado nas três safras (Tabela 2, 3 e 4).

$\mathrm{O}$ arranjo espacial agregado para $T$. limbativentris, detectado nesse estudo, difere da distribuição espacial aleatória para a população migratória do inseto na fase vegetativa da cultura, observada por Costa e Link (1992). Essa diferença pode ser atribuída ao menor número de amostras e ao método de amostragem com rede-de-varredura, adotado por estes autores, o qual atualmente não é recomendado quando as plantas de arroz encontram-se na fase vegetativa (REUNIÃO, 2014).

Muitos estudos têm demostrado que a maioria de espécies de artrópodes apresentam arranjo espacial do tipo agregado. Em pesquisas com outras espécies da família Pentatomidae, o padrão espacial observado foi semelhante ao observado para T. limbativentris neste estudo. Souza et al. (2013), ao determinarem a distribuição espacial de Euschistus heros (Fabricius, 1974) (Hemiptera: Pentatomidae) na cultura da soja por meio de diferentes índices de 
dispersão, verificaram, predominantemente, um comportamento agregado para ninfas e adultos do percevejo. ReayJones et al. (2010), também verificou uma disposição espacial agregada, independentemente da fase de vida dos insetos, para as espécies Euschistus servus (Say, 1832) (Hemiptera: Pentatomidae), Oebalus pugnax (Fabricius, 1775) (Hemiptera: Pentatomidae), Nezara viridula (Linnaeus, 1758) (Hemiptera: Pentatomidae) e Thyanta custator (Fabricius, 1803) (Hemiptera: Pentatomidae), em trigo. Em lavouras comerciais de arroz, no sul da Flórida, Foster et al. (1989) também constataram distribuição espacial para ninfas, adultos e ninfas+adultos do percevejo-do-grão Oebalus pugnax (Fabricius, 1775) (Hemiptera: Pentatomidae).

O arranjo espacial agregado do percevejo-do-colmo indica que no monitoramento da população devem-se amostrar tanto os adultos como as ninfas (PAZINI et al., 2015), pois ambos causam danos à cultura e as ninfas, muitas vezes, representam a maioria da população. Também, incluem-se as ninfas de primeiro instar que, mesmo não se alimentando, representam o potencial de crescimento da população em poucas semanas.

No âmbito do MIP em arroz irrigado, a validação do arranjo espacial de T. limbativentris, obtida nesse estudo, é fundamental para elaboração de um plano de amostragem mais eficiente para o inseto em lavouras comerciais de arroz, proporcionando maior segurança na tomada de decisão de controle, ainda mais se aliada ao conhecimento do nível de dano econômico ocasionado pelo inseto em diferentes fases de desenvolvimento das plantas. Além disso, essa informação poderá contribuir para a redução do emprego de inseticidas, com restrição para somente em épocas e áreas com verdadeira necessidade de controle, como em zonas de agregação (focos do inseto) no interior da lavoura.

\section{Considerações finais}

O arranjo espacial de Tibraca limbativentris, independente do nível de infestação e fase de vida do inseto, é do tipo agregado, validado pelos índices: razão variância/média, índice de Morisita, índice k, coeficiente de Green e potência de Taylor, em três safras consecutivas de arroz irrigado por inundação na região do Planalto da Campanha do Rio Grande do Sul.

\section{Agradecimentos}

À Embrapa Clima Temperado pela concessão de bolsas de estudos aos envolvidos no projeto e à equipe técnica da Fazenda Pitangueira - Unidade Cambaí por disponibilizar a área do estudo.

\section{Referências}

ALVES TM, MAIA AHN, BARRIGOSSI JAF. Spatial distribution and coexisting patterns of adults and nymphs of Tibraca limbativentris (Hemiptera: Pentatomidae) in paddy rice fields. Environ. Entomol. 2016;45(6):15051514.

BOTTA RA, SILVA FF da, PAZINI J de B, MARTINS JF da S, RÜBENICH. Abundância sazonal de percevejo-docolmo do arroz. Pesq. Agropec. Trop. 2014;44(4):417-423.

BOTTON M, MARTINS JF da S, LOECK AE, ROSENTHAL MD. Biology of Tibraca limbativentris Stal on rice plants. An. Soc. Entomol. Bras. 1996;25(1):21-26. 
CHIBA MK, GUEDES FILHO O, VIEIRA SR. Variabilidade espacial e temporal de plantas daninhas em Latossolo Vermelho argiloso sob semeadura direta. Acta. Sci. Agron. 2010;32(4):735-742.

CONAB [Internet]. Brasília: Companhia Nacional de Abastecimento [cited 2015 jan 07]. Acompanhamento de safra brasileira: grãos, décimo segundo levantamento, setembro 2015. Available from: http://www.conab. gov.br/OlalaCMS/uploads/arquivos/15_09_11_10_42_03_boletim_graos_setembro_2015.pdf.

COSTA EC, LINK D. Dispersão de Tibraca limbativentris Stal, 1860 (Hemiptera: Pentatomidae) em arroz irrigado. An. Soc. Entomol. Bras. 1992;21(1):197-202.

COSTA MG, BARBOSA JC, YAMAMOTO PT. Distribuição de probabilidade de ocorrência de Orthezia praelonga Douglas (Hemiptera: Sternorrhyncha: Ortheziidae) na cultura de citros. Neotrop. Entomol. 2006;35(3):395-401.

COSTA MG, BARBOSA JC, YAMAMOTO PT. Planos de amostragem seqüenciais para Orthezia praelonga Douglas (Hemiptera: Sternorrhyncha, Ortheziidae) na cultura de citros. Neotrop. Entomol. 2007;36(6):932938.

COSTA MG, BARBOSA JC, YAMAMOTO PT, LEAL RM. Spatial distribution of Diaphorina citri Kuwayama (Hemiptera: Psyllidae) in citrus orchards. Sci. agric. 2010;67(5):546-554.

COUNCE PA, KEISLING C, MITCHELL AJ. A uniform, objective, and adaptative system for expressing rice development. Crop. Sci. 2000;40(2):436-443.

DINARDO-MIRANDA LL, VASCONCELOS ACM, VIEIRA SR, FRACASSO JV, GREGO CR. Uso de geoestatística na avaliação da distribuição espacial de Mahanarva fimbriolata em cana-de-açúcar. Bragantia 2007;66(3):449-455.

ELLIOTT JM. Some methods for the statistical analysis of sample benthic invertebrates. 2ed. Ambleside: Freshwater Biological Association; 1979. 157p.

FOSTER RE, CHERRY RH, JONES DB. Spatial distribution of the rice stink bug (Heteroptera: Pentatomidae) in Florida rice. J. Econ. Entomol. 1989;82(2):507-509.

GREEN RH. Measurement of non-randomness in spatial distributions. Res. Popul. Ecol. 1966;8(1):1-7.

MARTINS GLM, VIEIRA MR, BARBOSA JC, DINI TA, MANZANO AM, ALVES BMS, SILVA RM da. Distribuição espacial de Calacarus heveae feres na cultura da seringueira em Marinópolis - São Paulo. Rev. Árvore 2012;36(2):211-217.

MELO EP de, FERNANDES MG, DEGRANDE PE, CESSA RMA, SALOMÃO JL, NOGUEIRA RF. Distribuição espacial de plantas infestadas por Spodoptera frugiperda (J.E. Smith) (Lepidoptera: Noctuidae) na cultura do milho. Neotrop. Entomol. 2006;35(5):689-697.

MYERS JH. Selecting a measure of dispersion. Environ. Entomol. 1978;7(5):619-621.

NICKELE MA, OLIVEIRA EB de, REIS FILHO W, IEDE ET, RIBEIRO RD. Distribuição espacial de formigueiros de Acromyrmex crassispinus (Forel) (Hymenoptera: Formicidae) em plantios de Pinus taeda. Neotrop. Entomol. 2010;39(6):862-872.

OKSANEN JF, BLANCHET G, FRIENDLY M, KINDT R, LEGENDRE P, MCGLINN D, MINCHIN PR, O'HARA RB, SIMPSON GL, SOLYMOS P, STEVENS MHH, SZOECS E, WAGNER H. Vegan: community ecology package. R package version 2.3-0. 2015. [cited 2015 aug 17]. Available from: http://cran.r-project.org/ web/packages/vegan/vegan.pdf.

PAZINI J de B, BOTTA RA, SILVA FF da. Mortalidade de percevejo-do-colmo do arroz no preparo do solo para cultivo mínimo. Pesq. agropec. bras. 2012;47(7):1022-1024. 
PAZINI J de B, BOTTA RA, SEIDEL EJ, SILVA FF da, MARTINS JF da S, BARRIGOSSI JAF, RÜBENICH R. Geoestatística aplicada ao estudo da distribuição espacial de Tibraca limbativentris em arrozal irrigado por inundação. Cienc. Rural 2015;45(6):1006-1012.

R DEVELOPMENT CORE TEAM. R - A language and environment for statistical computing. ver. 2.15.0. Vienna, Austria: R Foundation for Statistical Computing. 2011. [cited 2015 aug 17]. Available from: www.Rproject.org.

RABINOVICH JE. Introducción a la ecología de poblaciones animales. México: Compania editorial continental; 1980. 313 p.

REUNIÃO TÉCNICA DA CULTURA DO ARROZ IRRIGADO, 30., 2014, Bento Gonçalves. Arroz irrigado: recomendações técnicas da pesquisa para o Sul do Brasil. Santa Maria: SOSBAI; 2014. 192p.

REAY-JONES FPF, TOEWS MD, GREENE JK, REEVES RB. Spatial dynamics of stink bugs (Hemiptera: Pentatomidae) and associated boll injury in southeastern cotton fields. Environ. Entomol. 2010;39(3):959969.

RIBEIRO R, PEREIRA MFA, MARTINELLI NM, MACCAGNAN DHB. Dispersão de Fidicinoides sp. (Hemiptera: Cicadidae) em cafeeiro. Científica 2006;34(2):263-268.

RODRIGUES TR, FERNANDES MG, SANTOS HR dos. Distribuição espacial de Aphis gossypii (Glover) (Hemiptera, Aphididae) e Bemisia tabaci (Gennadius) biótipo B (Hemiptera, Aleyrodidae) em algodoeiro Bt e não-Bt. Rev. Bras. entomol. 2010;54(1):136-143.

SILVA JF da, BLEICHER E, MARQUES GV, SILVA V. Distribuição espacial do pulgão preto em feijão de corda e cálculo do número de amostras. Rev. Ciencia Agron. 2014a;45(3):537-542.

SILVA V, MARQUES GV, SILVA JF da, BLEICHER E. Distribuição espacial da cigarrinha Empoasca kraemeri Ross \& Moore (Hemiptera: Cicadelidae) no feijão-de-corda e cálculo do número de amostras. Arq. Inst. Biol. 2014b;81(4):335-341.

SILVEIRA NETO S, NAKANO O, BARDIN D, VILLA NOVA NA. Manual de ecologia dos insetos. São Paulo: Agronômica Ceres; 1976. 419p.

SOUZA LA, BARBOSA JC, GRIGOLLI JFJ, FRAGA DF, MALDONADO JÚNIOR W, BUSOLI AC. Spatial Distribution of Euschistus heros (F.) (Hemiptera: Pentatomidae) in soybean. Neotrop. Entomol. 2013;42(4):412-418.

TAYLOR LR. Aggregation, variance and the mean. Nature 1961;189(4766):732-735.

TOLEDO F, BARBOSA JC, YAMAMOTO PT. Distribuição espacial de Toxoptera citricida (Kirkaldy) (Hemiptera: Aphididae) na cultura de citros. Rev. Bras. Frutic. 2006;28(2):194-198. 